\begin{tabular}{|l|l|c|c|}
\hline Eiszeitalter $u$. Gegenwart & 38 & $1-5$ & Hannover 1988 \\
\hline
\end{tabular}

\title{
Der Paul-Woldstedt-Stein - ein Findling auf der Rehburger Endmoräne am Steinhuder Meer / Hannover
}

\author{
ERnst-Rüdiger Look \& Klaus-Dieter Meyer *)
}

Am 14. Oktober 1988 wäre PAUL WOLDSTEDT (14. 10. 1888 - 7. 7. 1973) 100 Jahre alt geworden.

In Würdigung seiner menschlichen und wissenschaftlichen Persönlichkeit haben wir inmitten eines seiner ehemaligen Hauptarbeitsgebiete einen nordischen Findling zum

\section{Paul-Woldstedt-Stein}

widmen lassen.

Unweit nördlich des Steinhuder Meeres, innerhalb der Gemarkung Mardorf der Stadt Neustadt a. Rbge. (Landkreis Hannover) fand der Landwirt KARL KOOP bei einer Waldrodung südlich der Diepholzberge (s. Abb. 1) im Februar 1981 einen 4,2 $\times 1,7 \times 1,6 \mathrm{~m}$ großen Findling; das Gewicht wird auf rund 20-25 t geschätzt. Derartig große Findlinge sind im Altmoränengebiet der nordischen pleistozänen Eiszeiten, wie hier, selten anzutreffen. Nur der "Goliath-Stein” direkt nördlich des Steinhuder Meeres bei der Gaststätte "Weiße Düne”, östlich von Mardorf, ist ein etwa gleichgroßer Findling. Östlich von Nienburg bei der Ortschaft Stöckse ist mit dem „Giebichenstein” ein außergewöhnlich großer Findling bekannt, mit ca. $7 \times 5 \times 3,5 \mathrm{~m}$ der größte Niedersachsens.

Auch von seiner Beschaffenheit her ist der Block bemerkenswert. Es handelt sich nach der mikroskopischen Untersuchung (DS 26867) durch P. MüLLER (BGR Hannover) um einen guterhaltenen, rötlichgrauen Granit, der unter hohem Druck und erhöhter Temperatur eine deutliche flaserige Parallelstruktur erhielt, also auch als "Gneisgranit" bezeichnet werden kann. Derartige Gesteine sind vor allem in

*) Anschrift der Verfasser: Prof. Dr. E.-R. LooK; Prof. Dr. K.-D. MEYER, Niedersächsisches Landesamt für Bodenforschung, Stilleweg 2, 3000 Hannover 51.
Schweden beheimatet. Als Mindestalter sind über 1 Milliarde Jahre anzunehmen.

Dieser außergewöhnliche Findling wurde vor über 200.000 Jahren, während des Drenthe-Stadiums der Saale-( = vorletzte) Eiszeit, im Inlandgletschereis in Schweden eingefroren und bis an seine Fundstelle am Steinhuder Meer geschoben. Die Fundschicht war kiesiger Geschiebedecksand über Schmelzwassersand; nach Voss (1982) liegt er in einer Endmoränenlandschaft mit Resten von Grundmoräne und Dünen- und Flugsanddecken. Der jetzt gut 1.000 Kilometer von seinem Heimatort in Schweden entfernt liegende Findling weist einige Besonderheiten auf, die auf die „Reisestrapazen” zurückzuführen sind. Vom Schieben im Eis zeugen gerundete Ecken und eine glattgeschliffene Gleitfläche, in die deutlich erkennbar Rillen eingekritzt sind (Gletscherschrammen). Scharfe Kanten dürften auf Absprengungen durch Frostverwitterungen zurückgehen.

In der jüngsten, der Weichsel-Eiszeit, wurde der Findling in der eisfreien Landschaft zu einem Windkanter

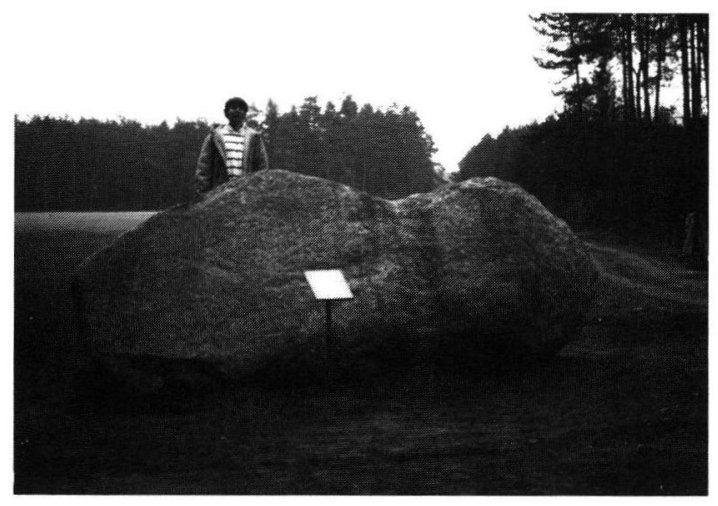

Abb. 1: Der Paul-Woldstedt-Stein am Steinhuder Meer, Gemarkung Mardorf, Landkreis Hannover

(Foto E.-R. LOOK). 
geformt: Unbehindert konnten sandbeladene Winde seine Oberfläche nicht nur abhobeln, sondern auch glattpolieren. Davon zeugt heute eine auffällige Kante auf der oberen Findlingsseite.

Jüngere Sandablagerungen haben ihn schließlich zugedeckt. Das kann mit Ursache dafür sein, daß dieser Findling im 19. Jahrhundert nicht zerstört wurde, als im Zuge des Straußenbaus viele andere Findlinge zu Pflastersteinen zerschlagen wurden.

Da die Zerstörung von Findlingen auch heute noch fortschreitet, haben wir zum Schutz besonders charakteristischer Gesteine und zu Lehr- und Anschauungszwecken bei Hagenburg am Steinhuder Meer einen „Findlingsgarten” eingerichtet (MEYER 1980).

Der Findling wurde vom Grundstückseigentümer, Herrn Landwirt KARL KOOP aus Mardorf, freigelegt. Mit schwerem Bergegerät des Panzerartilleriebataillon 35 aus Luthmersen konnte er von seinem Fundplatz (R 352278, H 581864) an seinen heutigen Standort an einer Wegegabel gebracht (s. Abb. 2) und dort richtig plaziert werden $(\mathrm{R}=352217, \mathrm{H}=581850)$. Im Juni 1982 wurde dieser Findling im Beisein von zahlreichen Personen des öffentlichen Lebens und interessierter Bürger vom Landrat, Herrn Dr. KARSTEN HOPPENSTEDT, zum Naturdenkmal erklärt und ge- meinsam mit dem Vizepräsidenten des Landesamtes für Bodenforschung, Herrn Prof. Dr. HEINRICH REXHÄUSER, mit Neustädter Sekt der Marke „Schloß Landestrost" getauft und PAUL WOLDSTEDT gewidmet. In der inzwischen dort angebrachten Widmungstafel ist folgendes zum Gedenken unseres nun 100jährigen Jubilars nachzulesen:

\section{Paul-Woldstedt-Stein}

Granitfindling eingefroren im Gletschereis und mit diesem aus Schweden hierher geschoben

Alter: über 1 Milliarde Jahre

Gewicht: ca. 20 Tonnen.

Zur Erinnerung an den Eiszeitforscher

Prof. Dr. PAUL WOLDSTEDT (1888-1973), der erstmalig im Jahre 1928 die Schneerener Berge nördlich vom Steinhuder Meer als Teil der Endmoräne der „Rehburger Phase” erkannte.

Dieser Endmoränenzug wurde während der Saale-Eiszeit vor ca. 200.000 Jahren durch das aus Skandinavien bis hierher vorgedrungene Inlandeis gebildet. Er erstreckt sich als Höhenzug nach Westen bis ins Emsland.

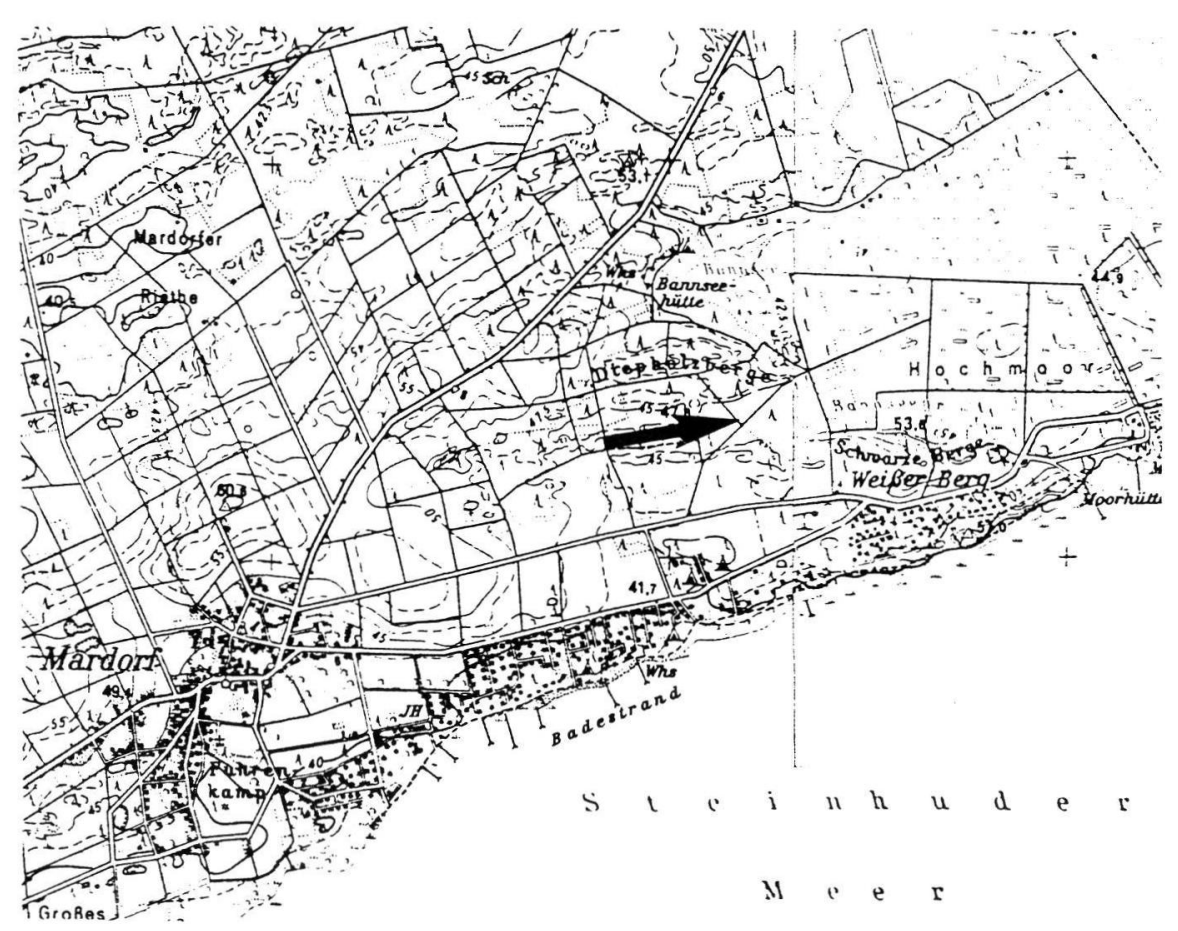

Abb. 2: Lageplan Paul-Woldstedt-Stein (siehe Pfeilspitze) am Steinhuder Meer / Hannover (Auszug TK 50, Nr. L 3520, L 3522). 
Erinnern wir uns auszugsweise an DUPHORN (1974) im Nachruf auf PAUL WOLDSTEDT: „Am 7. Juli 1973 verstarb PAUL WOLDSTEDT, der Nestor der deutschen Eiszeitgeologie, der auch im internationalen Maßstab zu den prominentesten Forschern gehörte. Welchen Klang sein Name in der internationalen Fachwelt hat, wurde anläßlich des Festkolloquiums zu seinem 80 . Geburtstag (1968) von einem ausländischen Kollegen halb ernst-, halb scherzhaft mit der Bemerkung zum Ausdruck gebracht, daß der Name WOLDSTEDT für viele synonom mit ,Eiszeit” sei.

PAUL WOLDSTEDT wurde am 14. Oktober 1888 in Flensburg geboren. Dort, im Lande der Förden und der „Buckligen Welt”, wie die Schleswig-Holsteiner die Jungmoränenlandschaft ihrer Heimat nennen, wuchs er gleichsam in die Materie des norddeutschen Vereisungsgebietes hinein. Im Jahre 1912 begründete er seine akademische Laufbahn mit einer Dissertation über „Beiträge zur Morphologie von Nordschleswig”. WOLDSTEDTs weltoffene Betrachtungsweise und seine Gabe der vergleichenden geologisch-geomorphologischen Zusammenstellung zeichnen sich bereits in der Dissertation ab. Doch zunächst sah es gar nicht so aus, als ob er sich ein Leben lang der Eiszeitforschung verschreiben würde. Nach dem 1 . Weltkrieg und einer kurzen Assistenzzeit am Geographischen Institut der Universität Halle trat er 1921 in die Preußische Geologische Landesanstalt Berlin ein. In den nachfolgenden Jahren kartierte er mehrere Blätter im Braunschweigischen. Dabei konzentrierte er sich mehr auf die Klärung der tektonischen und salztektonischen $\mathrm{Zu}$ sammenhänge als auf die quartären Ablagerungen und Formen.

Mit der ihm eigenen Beharrlichkeit begann P. WOLDSTEDT jedoch schon während dieser Kartierzeit, seine Kreise im Quartär des Norddeutschen Tieflandes immer weiter zu ziehen. Zunächst verfolgt er die in Nordschleswig erkannten Zusammenhänge zwischen den großen Sandern, Rinnenseen und subglaziären Schmelzwasserrinnen der letzten Eiszeit bis nach Ostpreußen weiter. Dann erschienen mehrere Arbeiten zur stratigraphischen Untergliederung des norddeutschen Pleistozäns, die im wesentlichen auch heute noch Gültigkeit besitzen." Wir fügen hier einen Auszug aus WOLDSTEDT's Arbeit ein, in der er den Begriff „Rehburger Phase" einführt:

\section{„Über einen wichtigen Endmoränenzug in Nordwestdeutschland}

von PAUL WoLdSTEDT in Berlin

\footnotetext{
. An dieser Stelle möchte ich auf einen wichtigen Endmoränenzug eingehen, der sich über größere Erstreckung hin verfolgen läßt.
}

Aus der Gegend N des Steinhuder Meeres hat 1908 H. SPETHMANN mehrere hintereinander gelegene Staffeln eines Endmoränenzuges beschrieben, die sich hier in WSW-ONO-Richtung erstrecken. Der Hauptzug ist der Schneerener Bogen, der vom Leiersberg N Rehburg in nordöstlicher Richtung bis in die Gegend von Mandelsloh im Leinetal verläuft. Ihm ist im Süden die Mardorfer Vorstaffel vorgelagert, während $\mathrm{N}$ des Hauptbogens noch die Husumer Nachstaffel folgt, finden die Endmoränen nach $\mathrm{O}$ hin ihre Fortsetzung in den Höhen von Abbensen und Mellendorf zwischen Leine und Wietze. An einen west-östlich streichenden Bogen (Brelinger Berg) schließt sich hier ein weiterer nach SO gerichteter an. Der Lindenberg auf der kleinen Diluvialhochfläche von Scharrel mag eine Vorstaffel dieses Hauptzuges darstellen und ist vielleicht mit der Mardorfer Staffel in Verbindung zu bringen.

Zwischen Fuhse und Oker ist ein Zug von Kieskuppen vorhanden, die, je weiter nach O hin, um so deutlicher im Gelände hervortreten. Sie sind auch heute noch in ihrer äußeren Form deutlich hervortretende Endmoränenkuppen.

Betrachten wir die Fortsetzung der Rehburger Moräne nach W hin, so findet die südliche Staffel, die Mardorfer, eine Fortsetzung in der Loccumer Endmoräne, die bei Ilvese am Weserufer abbricht. Die Fortsetzung ist in den flachen NNW streichenden Erhebungen zwischen Harrienstedt und Uchte und N Uchte zu suchen. Hier vereinigt sich diese südliche Staffel mit einer nördlichen, die die ausgeprägte Moränenlandschaft zwischen Kuppendorf und Hoysinghausen bildet und durch den Knapp-Berg und Heisterberg (N Steyerberg) mit der Husumer Staffel in Zusammenhang steht.

Von Kuppendorf verläuft die Moräne, vielfach durch breite Täler unterbrochen, in westlicher Richtung über Göthen Holzhausen - Hann. Ströhen - Wagenfeld zu der Kellenberg-Moräne O Diepholz. Durch den Hohen Sühn ist dann weiter die Verbindung zu den Dammer Bergen gegeben.

Diese sind einige der wichtigsten und ausgeprägtesten Glieder des ganzen Zuges; sie bilden eine markante Erhebung, die von Vechta in Oldenburg in SSW-Richtung auf Neuenkirchen zu verläuft, in ihren höchsten Kuppen $181 \mathrm{~m}$ erreicht und sich damit mehr als $100 \mathrm{~m}$ über die nähere Umgebung erhebt.

Durch das breite Hasetal von den Dammer Bergen getrennt, schließt sich nach Westen der breite Komplex der Fürstenauer Berge an, der das Gegenstück der Dammer Berge bildet und ihnen in Aufbau und Oberflächengestaltung völlig an die Seite zu stellen ist. Die weitere Fortsetzung des ausgeprägten Zuges ist über die Fenster-Berge NW Fürstenau, den Windmühlenberg N Thuine und die Erhebungen S und SW Baccum zu suchen. Es folgt dann die breite Unterbrechung durch das Emstal. Möglicherweise bilden die Erhebungen von Ootmarsum und Ülsen im preußisch-holländischen Grenzgebiet die weitere Fortsetzung dieses wichtigen Endmoränenzuges.

In der beschriebenen Eisrandlage haben wir wohl die wichtigste und ausgeprägteste Endmoräne Nordwestdeutschlands vor uns, die sich heute schon mit ziemlicher Sicherheit über etwa $300 \mathrm{~km}$ hin verfolgen läßt. Nach dem etwa in der Mitte 
des ganzen Zuges gelegenen Orte Rehburg bezeichne ich die Endmoräne als „Rehburger Endmoräne” und die dadurch bezeichnete Stillstandslage als , $\mathrm{R}$ e h b u r g e r S t a d i u m". . . ." (Woldstedt 1928).

„Unterdessen arbeitete er nebenbei an seinem Buch „Das Eiszeitalter. Grundlinien einer Geologie des Diluviums". Dieses Buch, das 1929 erschien, brachte ihm weltweite Anerkennung ein.

Mit dem internationalen Durchbruch mehrten sich auch die Auslandsreisen. Bereits 1928 hatte er eine mehrmonatige Studienreise in die nordamerikanischen Vereisungsgebiete durchgeführt. Seither hat er nie an der Gleichzeitigkeit der weltweiten pleistozänen Klimaverschlechterungen und Vereisungen gezweifelt. Auf dem 2. INQUA-Kongreß in Leningrad 1932 wurde er in das Redaktionskomitee der „Kommission für die Internationale Quartärkarte von Europa 1:1,5 Mio." gewählt. 1935 erschien seine "Geologisch-morphologische Übersichtskarte des norddeutschen Vereisungsgebietes 1:1,5 Mio.", die auch zur Wandkarte für den Schul- und Hochschulunterricht umgeändert wurde. Im Sommer 1936 folgte dann eine Studienreise nach Island. Im Vorland der isländischen Inlandeisgletscher studierte er die Toteisvorgänge in situ. Auf dieser aktuo-geologischen Grundlage baute er dann seine Untersuchungen über die Rolle des Toteises für die Glazialmorphologie Norddeutschlands auf.

Im 2. Weltkrieg führte P. WOLDSTEDT seine Kartierarbeiten in Norddeutschland weiter. Während dieser Zeit begann er auch mit der Bearbeitung seines Buches „Norddeutschland und angrenzende Gebiete im Eiszeitalter", das dann 1950 in der ersten und 1955 in der zweiten Auflage erschien. Dieses Buch, das nicht nur das Pleistozän und Holozän, sondern auch die menschlichen Kulturen in Norddeutschland behandelt, zeichnet sich dadurch aus, daß es die Information nicht nur auf den gesicherten Bestand des damaligen Wissens beschränkt, sondern daß es in vorsichtiger, abgewogener Beurteilung auch die ungeklärten und problematischen Fragen herausstellt.

Nach dem Kriege bemühte sich P. WOLDSTEDT, der inzwischen Abteilungsdirektor im Niedersächsischen Landesamt für Bodenforschung Hannover geworden war, um die Reorganisation der deutschen Quartärforschung und um den Wiederaufbau und Ausbau der internationalen Beziehungen. Dabei kamen ihm seine guten persönlichen Beziehungen aus der Vorkriegszeit sehr zustatten. In Hannover scharte er eine ganze Reihe von Quartärforschern um sich, und im Dezember 1947 rief er zusammen mit R. GRAHMANN und K. RiCHTER zur Gründung der Deutschen Quartärvereinigung auf. Die erste Tagung der DEUQUA fand im Oktober 1948 in Hannover statt. Auch die
Gründung der DEUQUA-Zeitschrift „Eiszeitalter und Gegenwart” im Jahre 1951 geht wesentlich auf P. WOLDSTEDT's Initiative zurück, der selber längere Zeit Vorsitzender der Vereinigung war. Bis 1967 war er Schriftleiter der DEUQUA-Zeitschrift. Diese enthält auch eine ganze Reihe von thematisch sehr breit gefächerten Beiträgen des Autors WOLDSTEDT.

Nach seiner Pensionierung im Jahre 1951 siedelte P. WOLDSTEDT von Hannover nach Bonn über. Dort widmete er sich als Honorarprofessor an der Universität Bonn ganz seinen Studenten und seinen wissenschaftlichen Interessen. 1954 erschien der Band I seines Buches „Das Eiszeitalter”, 1958 folgt der Band II, 1965 der Band III und 1969 der Band „Quartär” im Handbuch der Stratigraphischen Geologie.

Auf dem 6. INQUA-Kongreß in Warschau 1961 wurde P. WOLDSTEDT zum Präsidenten der „Kommission für die Internationale Quartärkarte von Europa $1: 2,5$ Mio." gewählt. Bis 1967 hat er alle Sitzungen dieser Kommission geleitet, eine Aufgabe, die nicht nur umfassende Sachkenntnisse, sondern auch großes taktisches Geschick verlangte. Außerdem führte er noch mehrere Forschungsreisen in das Ausland durch. Noch im hohen Alter reiste er gemeinsam mit seiner Frau ein halbes Jahr lang durch Südafrika, Australien und Neuseeland, um die neueren Forschungsergebnisse aus diesen Ländern in den Band III seines Buches „Das Eiszeitalter” einarbeiten zu können.

Anerkennungen und Ehrungen für seine wissenschaftlichen Leistungen sind P. WOLDSTEDT reichlich zuteil geworden. 1943 nahm ihn die "Akademie der Naturforscher Leopoldina" als Mitglied auf. 1948 verlieh ihm die „Deutsche Geologische Gesellschaft" die Hans-Stille-Medaille und 1958 die DEUQUA die Albrecht-Penck-Medaille. Mehrere Festschriften wurden ihm gewidmet, und anläßlich seines 70., 75. und 80. Geburtstages wurden in der Universität Bonn unter reger Beteiligung in- und ausländischer Quartärforscher Festkolloquien veranstaltet. Anläßlich seines 75. Geburtstages erhielt er die Ehrendoktorwürde der Universität Rostock.

P. WOLDSTEDT hat mehrere Bücher über das Quartär in aller Welt geschrieben. Trotzdem hat er nie das Quartär des norddeutschen Vereisungsgebietes aus den Augen verloren. Das norddeutsche Quartär war ihm am meisten ans Herz gewachsen; hier war er zu Hause, hier hat er seine wissenschaftliche Laufbahn vor über sechs Jahrzehnten begonnen und hier hat er sie auch beendet: der Tod nahm ihm die Feder ein Jahr vor der Herausgabe der dritten Auflage seines Buches über „Norddeutschland und angrenzende Gebiete im Eiszeitalter" aus der Hand. P. WOLDSTEDT hat in der Quartärforschung tiefe Spuren hinterlassen, die noch lange nachwirken werden. 


\section{Schriftenverzeichnis}

Duphorn, K. (1974): Paul Woldstedt. - Geol. Jb., A 25: 3-12, 1 Foto; Hannover.

MEYER, K.-D. (1980): Findlingsgarten in Hagenburg am Steinhuder Meer eröffnet. - Der Geschiebesammler, 14(1): 1-4, 2 Abb.; Hamburg.

SPETHMANN, H. (1908): Glaziale Stillstandslagen im Gebiet der mittleren Weser. - Mitt. geogr. Ges. Lübeck, 2. Reihe, 22: 1-17, 1 Abb.; Lübeck.
Voss, H.-H. (1982): Erläuterungen zu Blatt Nr. 3421 Husum. - Geol. Karte Niedersachsen 1:25 000: 130 S., 13 Abb., 8 Tab., 8 Kt.; Hannover.

WoLDSTEDT, P. (1928): Über einen wichtigen Endmoränenzug in Nordwestdeutschland. - Jber. nieders. geol. Ver., 21: 10-17, 1 Abb.; Hannover.

Manuskript eingegangen am 30.1. 1988. 